\title{
Measurements and Computations of Internal Temperatures of the IGBT and the Diode Situated in the Common Case ${ }^{\dagger}$
}

\author{
Paweł Górecki * and Krzysztof Górecki it \\ Department of Marine Electronics, Gdynia Maritime University, Morska 83, 81-225 Gdynia, Poland; \\ k.gorecki@we.umg.edu.pl \\ * Correspondence: p.gorecki@we.umg.edu.pl \\ + This paper is an extended version of our paper published in Proceedings of the 2020 27th International \\ Conference on Mixed Design of Integrated Circuits and System (MIXDES), Wrocław, Poland, \\ 25-27 June 2020, pp. 133-138.
}

check for

updates

Citation: Górecki, P.; Górecki, K. Measurements and Computations of Internal Temperatures of the IGBT and the Diode Situated in the Common Case. Electronics 2021, 10, 210. https://doi.org/10.3390/ electronics10020210

Received: 2 December 2020

Accepted: 15 January 2021

Published: 18 January 2021

Publisher's Note: MDPI stays neutral with regard to jurisdictional claims in published maps and institutional affiliations.

Copyright: (c) 2021 by the authors. Licensee MDPI, Basel, Switzerland. This article is an open access article distributed under the terms and conditions of the Creative Commons Attribution (CC BY) license (https:// creativecommons.org/licenses/by/ $4.0 /)$.

\begin{abstract}
This article proposes effective methods of measurements and computations of internal temperature of the dies of the Insulted Gate Bipolar Transistor (IGBT) and the diode mounted in the common case. The nonlinear compact thermal model of the considered device is proposed. This model takes into account both self-heating phenomena in both dies and mutual thermal couplings between them. In the proposed model, the influence of the device internal temperature on self and transfer thermal resistances is taken into account. Methods of measurements of each self and transfer transient thermal impedances occurring in this model are described and factors influencing the measurement error of these methods are analysed. Some results illustrating thermal properties of the investigated devices including the IGBT and the antiparallel diode in the common case are shown and discussed. Computations illustrating the usefulness of the proposed compact thermal model are presented and compared to the results of measurements. It is proved that differences between internal temperature of both dies included in the TO-247 case can exceed even $15 \mathrm{~K}$.
\end{abstract}

Keywords: thermal models; measurements; modelling; IGBT; thermal phenomena; power semiconductor devices

\section{Introduction}

Many electronic and power electronic circuits include Insulated Gate Bipolar Transistors (IGBTs) [1-4]. Properties of these devices strongly depend on their internal temperature $T_{j}$ [5-7]. This temperature exceeds ambient temperature $T_{a}$ due to thermal phenomena $[5,8,9]$. An increase in $T_{j}$ temperature causes shortening of lifetime of semiconductor devices and changes in values of their exploitation parameters [10-12].

Two semiconductor dies are mounted in the common case. For example, many dies of the IGBT are mounted together with the die of an anti-parallel diode. In such a case, self-heating occurs in each of the mentioned dies and additionally there are mutual thermal couplings between the two dies [13]. Figure 1 illustrates the schematic diagram of such a device (Figure 1a) and a simple schematic mounting manner of the mentioned dies in the common case (Figure $1 b$ ). As is visible, diode $D_{1}$ is connected between the collector and the emitter of transistor $\mathrm{T}_{1}$. Both dies are soldered to $\mathrm{Cu}$ substrate, which makes it possible to improve heat removal generated in both dies. The area of the IGBT die is twice bigger than the area of the diode die. The distance between these dies is equal to $2 \mathrm{~mm}$. Electrical connections between these dies and between lead frames and the dies are performed using bond wires. The base plate is a part of the device case.

To compute internal temperature of the semiconductor device when the value of power dissipated in this device is fixed, it is indispensable to use a thermal model of this device [14,15]. Compact thermal models are most frequently used [5,8,13,16,17]. Typically, the network representation of such a model is used to describe thermal properties of 
semiconductor devices. A compact thermal model of a semiconductor device contains such thermal parameters as thermal resistances $R_{t h}$ and thermal capacitances $C_{t h}$, which are estimated using the measured waveforms of transient thermal impedances $Z_{t h}(t)$. To take into account the influence of the device junction temperature on cooling efficiency, nonlinear thermal models should be used [18].

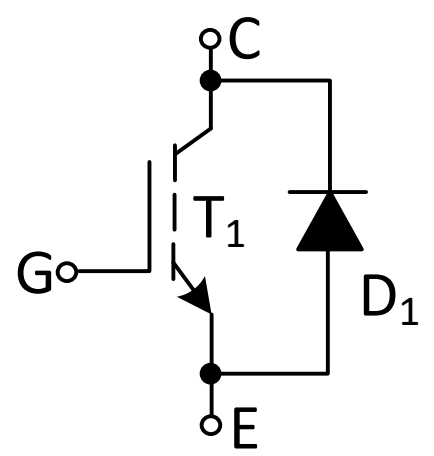

(a)

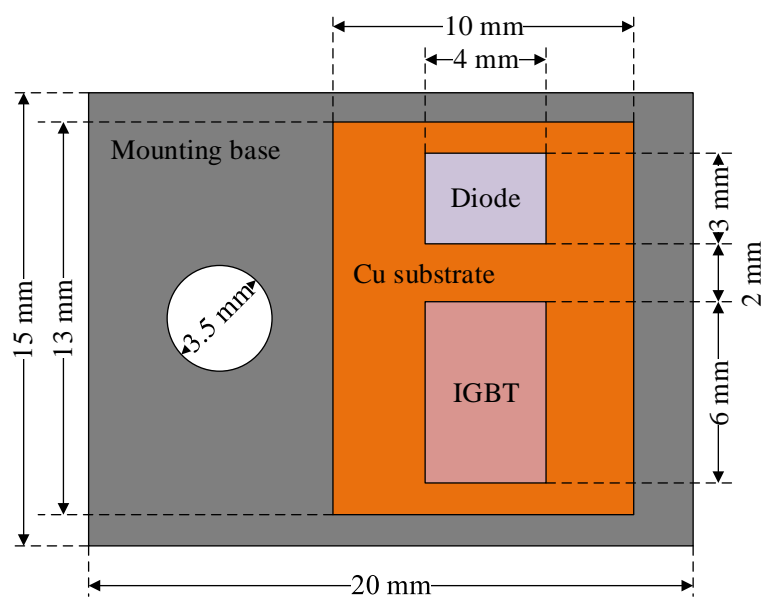

(b)

Figure 1. Schematic diagram of the investigated device (a); and a mounting manner of this device (b).

In the formulas defining transient thermal impedance of the device, internal temperature $T_{j}$, ambient temperature $T_{a}$ and dissipated power p exist. Values of temperature $T_{a}$ and power $\mathrm{p}$ can be measured in an easy way, whereas the value of temperature $T_{j}$ cannot be measured directly. The value of this temperature is measured indirectly with the use of optical or electrical methods $[19,20]$. With the use of optical methods, it is possible to measure the temperature of the semiconductor die only when laboratory semiconductor devices are not in the case. In contrast, for commercially made devices, optical methods make it possible only to measure the case temperature $T_{C}[18]$, which is lower than the device internal temperature.

On the other hand, indirect electrical methods allow measuring the device internal temperature on the basis of measurements of selected electrical parameters univocally dependent on temperature. Such parameters are called thermo-sensitive parameters $[13,16,17,21-23]$. The literature [17,21-23] describes possibilities of using different thermo-sensitive parameters to measure internal temperature of different semiconductor devices. For IGBTs, the voltage between the gate and the emitter of the transistor operating in the active range, the voltage between the collector and the emitter on the switched-on transistor or the voltage on the forward biased anti-parallel diode is used [22]. According to the JEDEC standard [24], the recommended thermo-sensitive parameter is the voltage between the collector and the emitter on the switched-on transistor.

In all the considered measurement methods and in compact thermal models of IGBTs $[5,17,21-23,25]$, only one internal temperature of this device is used. This temperature characterises thermal properties of the whole considered device.

An analysis of differences between values of internal temperature of the power Metal Oxide Semiconductor (MOS) transistor obtained with the use of indirect electrical methods, in which voltage on the forward biased $p-n$ junction or threshold voltage of the transistor are thermo-sensitive parameters, is presented in [23]. The problem of nonlinearity of thermometric characteristics while using threshold voltage as a thermo-sensitive parameter is discussed. On the basis of the results of simulations in the ANSYS software, it is shown that differences in the temperature value on the surface of the semiconductor structure of the area equal to $49 \mathrm{~mm}^{2}$ could reach even $50 \mathrm{~K}$. On the other hand, based on measurements with the electrical method and both thermo-sensitive parameters, it was observed that differences in the value of the transistor internal temperature are in the range of 4-11 K. 
The authors of [17] analysed the usefulness of selected thermo-sensitive parameters to measure internal temperature of power semiconductor devices. It is shown that the voltage on the forward biased p-n junction, through which current of a small value flows, is the most universal of the considered thermo-sensitive parameters.

Properties of measuring set-ups using different thermo-sensitive parameters of the IGBT are analysed in [22,23]. However, the fact that the diode and the transistor are situated in the common case, although they are separate semiconductor structures, is not taken into account [26].

In the catalogue data of the considered class of IGBTs, the following thermal resistances are given [26]: maximum thermal resistance junction-to-case of the IGBT $\left(R_{\text {thj-cIGBT }}\right)$, maximum thermal resistance junction-to-case of the diode $\left(R_{t h j-c D}\right)$, typical thermal resistance case-to-sink $\left(R_{t h C S}\right)$ and maximum thermal resistance junction-to-ambient $\left(R_{t h j-a}\right)$. In the catalogue data, the waveform of transient thermal impedance junction-to-case of the considered device is also given. However, the producer does not give any information about thermal parameters characterising the heat transfer between the case of the investigated device and the surroundings, because they depend on the applied cooling system of the investigated device.

As shown by the presented literature review, there is a lack of a nonlinear compact thermal model of the IGBT assembled together with the diode in the common case. Additionally, the problem of accurate measurements of internal temperatures of both dies contained in the considered devices is not studied.

In this paper, which is an extended version of the one presented in [27], two scientific problems are considered. The first problem is the method of effective computations and measurements of internal temperatures of the IGBT and diode dies contained in the common case. To solve this problem, a nonlinear compact thermal model of such devices is proposed. The second problem is the effective method of determining parameters occurring in this model. Such a method is proposed and it uses the results of measurements of self and transfer transient thermal impedances as the input data. Additionally, the influence of the choice of a thermo-sensitive parameter on the results of measurements of the mentioned thermal parameters of the investigated device is considered. Measuring set-ups making it possible to measure thermal parameters of this model with the use of different thermosensitive parameters are analysed. The results of measurements of thermal parameters occurring in the thermal model and the measurements performed with the use of the considered thermo-sensitive parameters for a selected IGBT operating at different cooling conditions are compared. These measurements are performed at transients and at the steady state. The results obtained at the steady state are also compared to the results of measurements performed with the use of the optical method. The obtained results and differences in temperatures of both dies included in the common case are discussed. In comparison to the conference paper [27], this article includes: the extended literature review, the new nonlinear compact thermal model of the investigated device, extended description of used measurement methods, results of measurements of self and transfer transient thermal impedances occurring in the elaborated model and comparison of the results of measurements to the results of computations performed with the use of the elaborated model.

\section{Nonlinear Compact Thermal Model of the Investigated Device}

In this paper, the IGBT containing two semiconductor dies in the common case: the transistor and the anti-parallel diode [26] is considered. Since these dies are situated in the common case, internal temperature of each of them depends on self-heating phenomena and mutual thermal couplings between these dies [13,28].

To determine the value of internal temperature of each mentioned semiconductor die at the well-known power dissipated in each of them, a thermal model is used [13,16,18,29,30]. One of the forms of such a model can be a network representation. In the literature, two typical networks representations of the compact thermal models are described: the Foster 
network and the Cauer network [31,32]. As stated in some papers (e.g., [31,32]), the Foster network does not have any physical interpretation, whereas the Cauer network can be obtained as a result of discretisation of the heat transfer equation. The classical form of the mentioned networks makes it possible to compute the device internal temperature with self-heating phenomena only. Additionally, in these networks, linear RC components are used and they do not make it possible to take into account the observed experimentally [18] dependences of efficiency of heat removal on the device internal temperature.

In this section, the nonlinear compact thermal model of the considered device is proposed. This model makes it possible to compute internal temperature of the IGBT and the diode taking into account self-heating in both devices and mutual thermal coupling between them. In Figure 2, the network representation of the formulated model is shown.

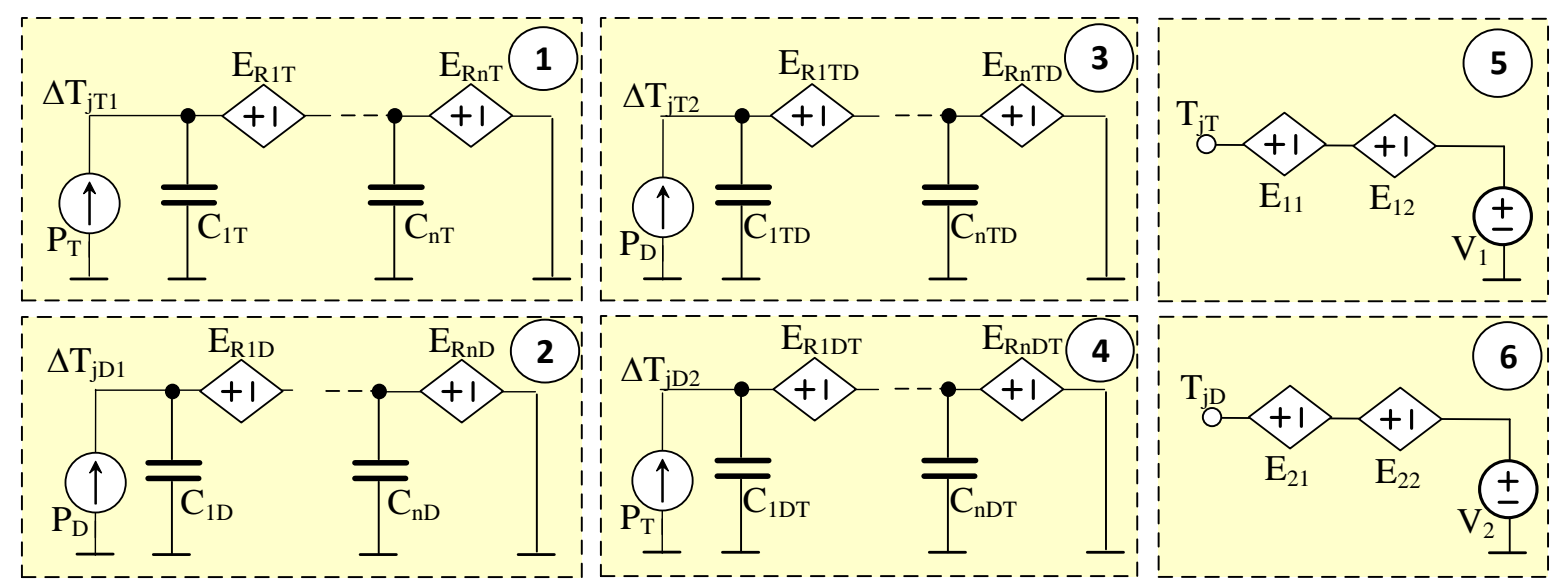

Figure 2. Network representation of the compact thermal model of the IGBT mounted in the same case with the antiparallel diode.

In the proposed thermal model values of internal temperatures of the IGBT $\left(T_{j T}\right)$ and the diode $\left(T_{j D}\right)$ are computed taking into account self-heating phenomena in each of these devices and mutual thermal couplings between them. In the network presented in Figure 1, six subcircuits (marked with natural numbers from 1 to 6 ) are visible.

Subcircuits 1 and 2 situated on the left-hand side of the figure are used to compute the value of an excess of internal temperatures of the transistor $\Delta T_{j T 1}$ and the diode $\Delta T_{j D 1}$ caused by self-heating phenomena in each of these devices. In these subcircuits, current sources represent power dissipated in the transistor $\left(P_{T}\right)$ and in the diode $\left(P_{D}\right)$. Capacitors $\mathrm{C}_{1 \mathrm{~T}}, \mathrm{C}_{\mathrm{nT}}, \mathrm{C}_{1 \mathrm{D}}$ and $\mathrm{C}_{\mathrm{nD}}$ model thermal capacitances, whereas controlled voltage sources $E_{R 1 T}, E_{R n T}, E_{R 1 D}$ and $E_{R n D}$ represent thermal resistances depending on internal temperature of the dies. The mentioned components model self transient thermal impedances of the transistor $Z_{t h T}(t)$ and the diode $Z_{t h D}(t)$.

The next two subcircuits ( 3 and 4 ) are used to describe mutual thermal couplings between the diode and the transistor. An increase in internal temperatures of these devices is equal to $\Delta T_{j T 2}$ and $\Delta T_{j D 2}$, respectively. Networks containing capacitors and the controlled voltage sources occurring in the considered subcircuits represent transfer transient thermal impedance between the diode and the transistor $Z_{t h T D}(t)$.

In the mentioned subcircuits, the controlled voltage sources describe the dependences of self and transfer thermal resistances occurring in the presented model on internal temperature of the dies included in the modelled semiconductor device. These dependences have the same form. For example, the dependence of self thermal resistance of the transistor is as follows

$$
R_{t h T}=R_{t h T 0}+R_{t h T 1} \cdot \exp \left(-\frac{T_{j T}-T_{a}}{T_{Z}}\right)
$$


where $R_{t h T 0}$ denotes the minimum value of the considered thermal resistance, $T_{J T}$ is the internal temperature of the transistor, $T_{a}$ is the ambient temperature and $R_{t h T 1}$ and $T_{Z}$ are model parameters.

The dependences describing the output voltage of controlled voltage sources $E_{R 1 T}$, $\ldots, E_{R n T}$ are given by the formula

$$
E_{R i T}=i_{R i T} \cdot a_{i} \cdot R_{t h T}
$$

where $i$ is the natural number in the range from 1 to $n, i_{R i T}$ denotes current flowing through the voltage source $E_{R i T}$ and $a_{i}$ is the factor equal to the quotient of the part of thermal resistance occurring in $i$ th segment of the Cauer network and thermal resistance. The sum of all $a_{i}$ factors is equal to 1 .

In formulas describing self thermal resistance of the diode $R_{t h D}$ and transfer thermal resistance between the diode and the transistor $R_{t h T D}$ instead of temperature $T_{j T}$, internal temperature of the diode $T_{j D}$ occurs.

Internal temperatures of the diode $T_{j D}$ and the transistor $T_{j T}$ are computed in subcircuits 5 and 6 shown on the right side of Figure 1 . These temperatures are equal to the sum of ambient temperature $T_{a}$ represented by voltage sources $V_{1}$ and $V_{2}$, the output voltages of controlled voltage sources $E_{11}$ and $E_{21}$ representing self-heating phenomena and the output voltages of controlled voltage sources $E_{12}$ and $E_{22}$ representing mutual thermal couplings. The output voltage of source $E_{11}$ is equal to voltage $\Delta T_{j T 1}$, whereas voltage $E_{21}$ is equal to voltage $\Delta T_{j D 1}$. In turn, the output voltages of sources $\mathrm{E}_{12}$ and $\mathrm{E}_{22}$ are equal to voltages $\Delta T_{j T 2}$ and $\Delta T_{j D 2}$, respectively.

All the used self and transfer transient thermal impedances take into account all the mechanisms of heat transfer between both dies and the surroundings.

To practically use the formulated thermal model, the values of parameters occurring in it must be known. The values of thermal capacitances and thermal resistances existing in this thermal model are estimated on the basis of the measured waveforms of self transient thermal impedances of the transistor and the diode and transfer transient thermal impedance between these devices. The manner of estimating the parameters of the RC Cauer network is described, e.g., in [33]. Thermal capacitances occurring in the proposed model are directly obtained using this manner. In turn, values of parameters occurring in Equation (1) and describing self and transfer thermal resistances are obtained by an approximation of the measured dependences of these thermal resistances on internal temperatures of the dies.

The waveforms of the mentioned above transient thermal impedances are measured using indirect electrical methods. These methods are described in the next section.

\section{Measurement Methods}

Indirect electrical methods need thermo-sensitive parameters and special measuring set-ups. In Figure 3, the diagram of the measurement set-up of transient thermal impedance $Z_{t h T}(t)$ using the gate-emitter voltage $V_{G E}$ as a thermo-sensitive parameter is shown. In turn, in Figure 4, the measurement set-up of transfer transient thermal impedance between the transistor and the diode $Z_{t h T D}(t)$ using voltage on the anti-parallel diode $V_{D}$ as a thermosensitive parameter is presented. Finally, Figure 5 presents the measurement set-up of transient thermal impedance of diode $Z_{t h D}(t)$. In all measurement set-ups, switches are realised using power MOSFETs. 


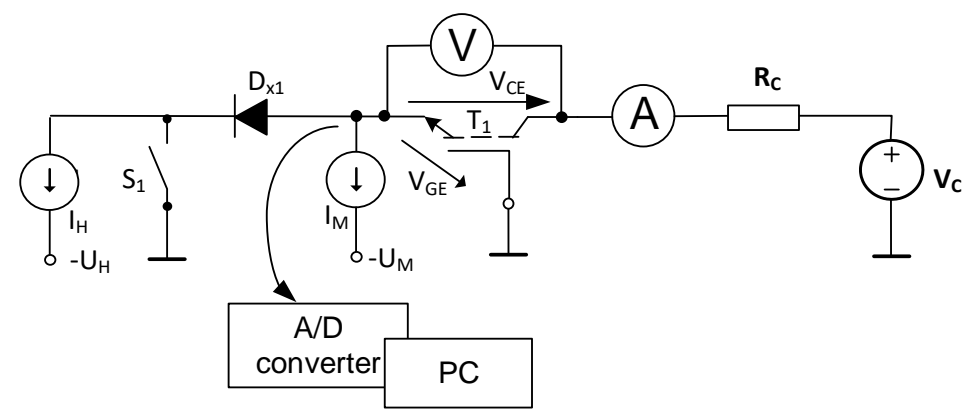

Figure 3. Measurement set-up to determine transient thermal impedance $Z_{t h T}(t)$ of the IGBT with the use of the gate-emitter voltage as a thermally sensitive parameter.

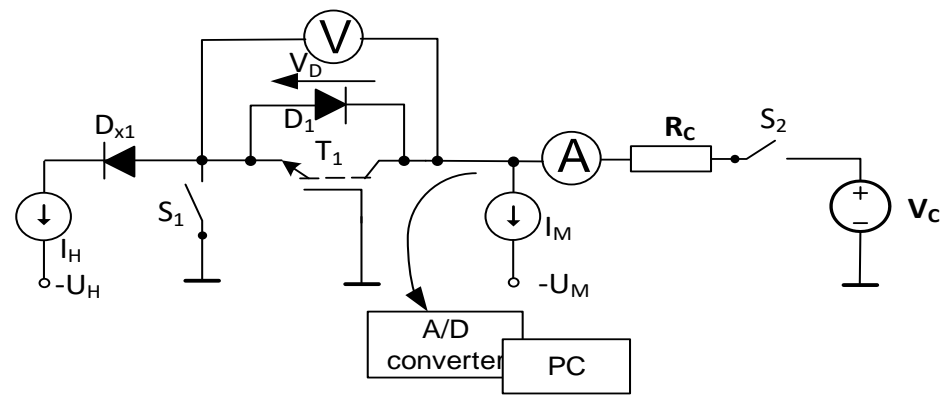

Figure 4. Measurement set-up to determine transfer transient thermal impedance $Z_{t h T D}(t)$ of the IGBT with the use of forward voltage of the anti-parallel diode as a thermally sensitive parameter.

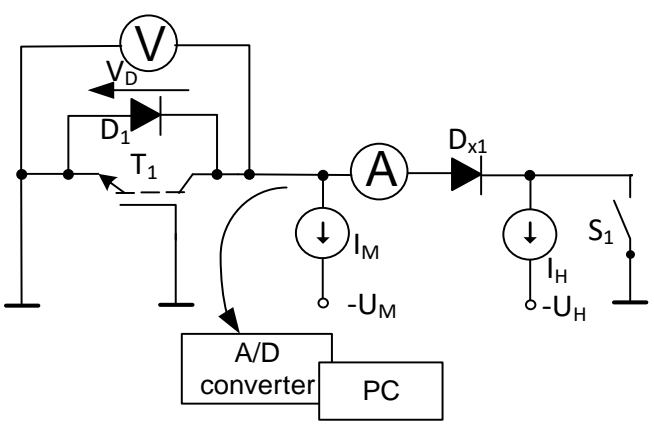

Figure 5. Measurement set-up to determine transient thermal impedance of the diode $Z_{t h D}(t)$ included in the investigated device.

In all considered measuring set-ups, proper transient thermal impedance is determined in three steps. The first step is a calibration of the thermometric characteristic describing the dependence of a thermo-sensitive parameter on temperature $\operatorname{TSP}(T)$. The calibration is performed at closed switches $S_{1}$ and opened switch $S_{2}$. In this step, through the investigated device flows current $\mathrm{I}_{\mathrm{M}}$ of a low value. During the calibration the temperature of the transistor is regulated by means of the thermostat, wherein the investigated device is situated. From this characteristic, the slope of the thermometric characteristic is computed or an analytic formula describing the dependence of the device internal temperature on a thermo-sensitive parameter is formulated.

In the second step of measurements, heating power is dissipated in the transistor operating within the active range (Figures 3 and 4 ) or in the forward biased diode (Figure 5), and its internal temperature increases as a result of self-heating phenomena. In this stage switches $S_{1}$ are opened, and switch $S_{2}$ is closed. Through the heating device (transistor or diode) flows current $I_{H}$. This step ends after obtaining the thermally steady state. At the steady state, the value of a voltage drop of the heating device $\left(V_{C E}\right.$ or $\left.V_{D}\right)$ is measured. The value of heating power $P_{H}$ is equal to the product of a voltage drop of the heating device and current $I_{H}$. 
The third step begins after a change of the state of switches at time $t=0$ and consists in measuring the waveform of the thermo-sensitive parameter until the steady state is obtained. Then, through the thermo-sensitive device (transistor or diode) flows current of the same value as during the calibration. The values of thermo-sensitive parameter TSEP $_{H}$ are measured in selected moments in time by means of the analogue-to-digital (A/D) converter and they are recorded in the computer. To stabilise the value of ambient temperature $T_{a}$ during heating and cooling, the investigated device is situated in the thermostat. The value of this temperature is measured with the use of an electronic thermometer with a thermoresistor.

With the use of the thermometric characteristic TSP $(T)$ measured during the calibrations and the waveform of the thermo-sensitive parameter TSEP $P_{H}(t)$ measured in the third step of measurements, the waveform of internal temperature $T_{j}(t)$ of the thermo-sensitive device is computed. Next, proper transient thermal impedance is computed using the following formula

$$
Z_{t h}(t)=\frac{T_{j}(t)-T_{a}}{P_{H}}
$$

The value of $Z_{t h}(t)$ obtained at the steady state is equal to thermal resistance $R_{t h}$. If transient thermal impedance of the transistor $Z_{t h T}(t)$ is measured, the waveform of temperature $T_{j T}(t)$ is used in Equation (3) and power $P_{H}$ is equal to the product of the collector current and $V_{C E}$ voltage. In turn, for transient thermal impedance of the diode $\mathrm{Z}_{\mathrm{thD}}(\mathrm{t})$, the waveform of temperature $T_{j D}(t)$ is used in Equation (3) and power $P_{H}$ is equal to the product of the diode current and $V_{D}$ voltage. Finally, for mutual transient thermal impedance between the transistor and the diode $Z_{t h T D}(t)$, the waveform of temperature $T_{j D}(t)$ is used in Equation (3) and power $P_{H}$ is equal to the product of the collector current and $V_{C E}$ voltage.

The investigations of the method were performed for the IRG4PC40UD device. For this transistor, thermometric characteristics were measured using the forward voltage of the anti-parallel diode, the gate-emitter voltage and the collector-emitter voltage as thermally sensitive parameters. In these measurements, the value of temperature is measured with the absolute error not higher than $\pm 1^{\circ} \mathrm{C}$ and the voltages are measured with the relative error below $0.1 \%$.

In [27], it is shown that, for low values of forward current of the anti-parallel diode, its thermometric characteristics are nonlinear. However, there is a range of the value of this current, for which the dependence $V_{D}(T)$ is linear. It is in the range from several hundred microamperes to several hundred milliamperes.

In Figure 6, thermometric characteristics of the anti-parallel diode are shown. As can be seen, these characteristics are practically linear and their slope changes from -2.6 to $-2.4 \mathrm{mV} / \mathrm{K}$.

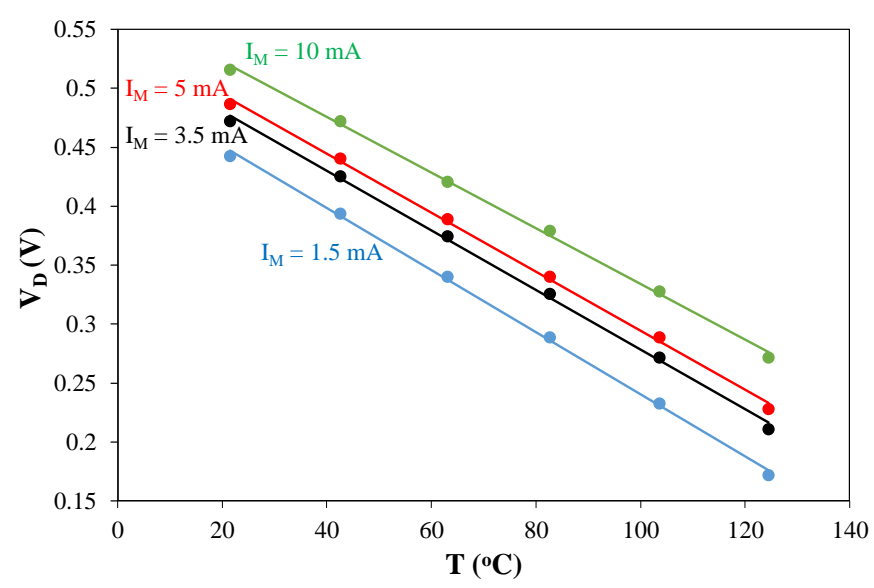

Figure 6. Thermometric characteristics of the anti-parallel diode for selected values of $\mathrm{I}_{\mathrm{M}}$ current. 
In turn, Figure 7 shows thermometric characteristics $V_{G E}(T)$ measured at selected values of measurement current $\mathrm{I}_{\mathrm{M}}$ and $V_{C E}$ voltage equal to $40 \mathrm{~V}$, which guarantees operation of the investigated transistor in the active mode. As is visible, these characteristics are nonlinear. They can be approximated with the use of the square function. For example, for $I_{M}=1 \mathrm{~mA}$, this formula has the following form:

$$
V_{G E}=5.4038 \mathrm{~V}-7.8 \frac{\mathrm{mV}}{{ }^{\circ} \mathrm{C}} \cdot T+10^{-5} \frac{\mathrm{V}}{\left({ }^{\circ} \mathrm{C}\right)^{2}} \cdot T^{2}
$$

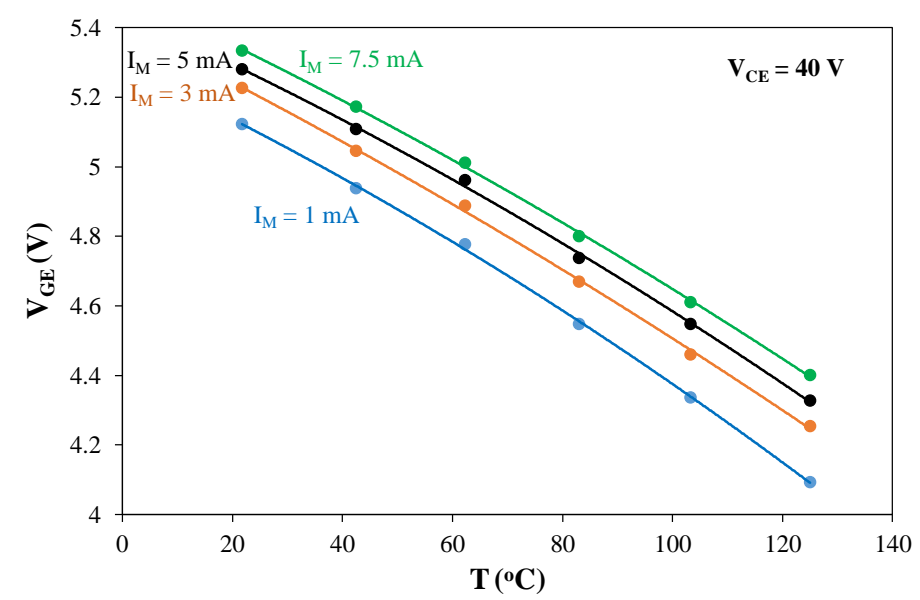

Figure 7. Thermometric characteristics $V_{G E}(T)$ for selected values of the collector current.

As can be noticed, characteristics $V_{G E}(T)$ show considerably higher (almost four times) sensitivity of the thermo-sensitive parameter at changes in temperature than characteristics $V_{D}(T)$. This means that, at the same accuracy of measurements of both voltages, four times higher resolution of measurements of the device internal temperature using $\mathrm{V}_{\mathrm{GE}}$ voltage as a thermo-sensitive parameter can be obtained.

Figure 8 illustrates the dependences $V_{C E}(T)$ measured at selected values of $\mathrm{I}_{\mathrm{M}}$ current. The presented dependences were obtained at $\mathrm{V}_{\mathrm{GE}}$ voltage equal to $10 \mathrm{~V}$, which is much higher than the threshold voltage.

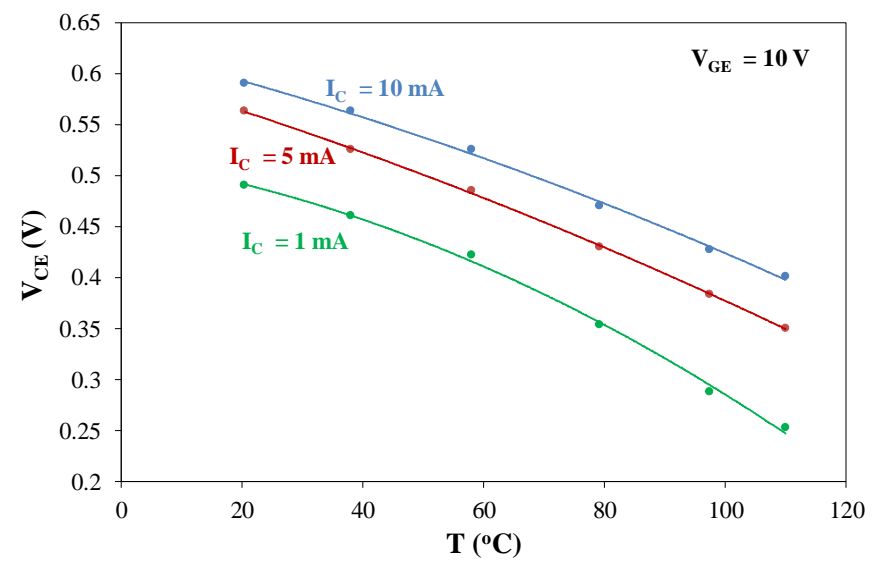

Figure 8. Thermometric characteristics $V_{C E}(T)$ for selected values of the collector current.

As is visible, the dependences $V_{C E}(T)$ are nonlinear for low values of the collector current $\left(I_{C}=1 \mathrm{~mA}\right)$ and nearly linear for much higher values of this current. The average value of the slope of these dependences belongs to the range from $-2.11 \mathrm{mV} / \mathrm{K}$ to $-2.67 \mathrm{mV} / \mathrm{K}$.

The accuracy of indirect electrical methods of measuring thermal parameters of the IGBT is analysed in $[27,34]$. Based on the considerations presented in the cited papers, 
the following analytical formula describing the relative error of measurements of thermal resistance $R_{t h T}$ can be obtained.

$$
\delta_{R t h T}=2 \cdot \frac{\Delta T S E P}{\operatorname{TSEP}_{H}(t=0)-T S E P_{K}}+\frac{\Delta \alpha_{T S E P}}{\alpha_{T S E P}}+\frac{\Delta I_{H}}{I_{H}}+\frac{\Delta V_{C E}}{V_{C E}}
$$

where $\triangle T S E P, \Delta \alpha_{T S E P}, \Delta I_{H}$ and $\Delta V_{C E}$ denote the absolute errors of measurements of the TSEP, the slope of the thermometric characteristic, the current $\mathrm{I}_{\mathrm{H}}$ and the collector-emitter voltage $V_{C E}$ at the end of the second step of measurements, respectively.

Next, the value of the TSEP is measured with the use of the fast A/D converter with the absolute measurement error equal to $2.5 \mathrm{mV}$. In turn, the error of estimating the slope of the thermometric characteristic is $\Delta \alpha_{\text {TSEP }} \leq 20 \mu \mathrm{V} / \mathrm{K}$ [34]. The difference of the value of $\operatorname{TSEP}_{H}(t=0)-T S E P_{K}$ depends on an increase of internal temperature of the considered transistor while heating it. For example, assuming that the considered increase is equal to $100 \mathrm{~K}$, the slope of the thermometric characteristic $\alpha_{T S E P}$ amounts to $-2 \mathrm{mV} / \mathrm{K}$, and the remaining errors accept the values given above; the minimum value of the error of measurements $R_{t h T}$ amounts to $3.7 \%$. If an increase of temperature is equal to only $20 \mathrm{~K}$, this error increases up to $13.7 \%$ [27]. The obtained values of the measurement error are close to the values of the measurement error of thermal parameters of IGBTs performed with the optical method and computed in $[27,34]$.

As shown in [34], the accuracy of the indirect electrical method of measurements of thermal resistance of the IGBT is the highest when an increase in junction temperature over ambient temperature is the highest and the slope of the thermometric characteristic is the biggest. Analysing the thermometric characteristic shown in Figures 6-8, it can be easily observed that the measurement error of $Z_{t h T}(t)$ is the smallest if $V_{G E}$ voltage is used as a thermo-sensitive parameter.

\section{Investigations Results}

To verify the usefulness of the proposed thermal model and the measurement method, some measurements and computations were performed. Investigations were performed for the IGBT of the type IRG4PC40UD by International Rectifier operating at different cooling conditions. This transistor is mounted in the TO-247 case and its parameters are described in [26]. The measurements were performed for this device situated on the aluminium-heat-sink of the dimensions $78 \mathrm{~mm} \times 70 \mathrm{~mm} \times 34 \mathrm{~mm}$ and for this transistor operating without any heat-sink.

The next subsections present the results of measurements of self and transfer transient thermal impedances characterising thermal properties of the investigated device and some results of measurements and computations illustrating the non-uniformity of temperature distribution inside the investigated device. The results of measurements obtained at the steady state were compared to the results of measurements obtained with the use of the optical method.

\subsection{Thermal Parameters}

With the use of the measurement methods described in Section 3, the measurements of waveforms of transient thermal impedances $Z_{t h T}(t), Z_{t h D}(t)$ and $Z_{t h T D}(t)$ of the investigated device operating at both types of the mentioned cooling conditions were performed.

With the use of the described measurement methods and the measured thermometric characteristics, waveforms of self transient thermal impedances of the transistor $Z_{t h T}(t)$ and the diode $Z_{t h D}(t)$ and transfer transient thermal impedance between the transistor and the diode $Z_{\text {thTD }}(t)$ were determined. These waveforms are presented in Figure 9 for the investigated device situated on the heat-sink and in Figure 10 for the device operating without any heat-sink. 


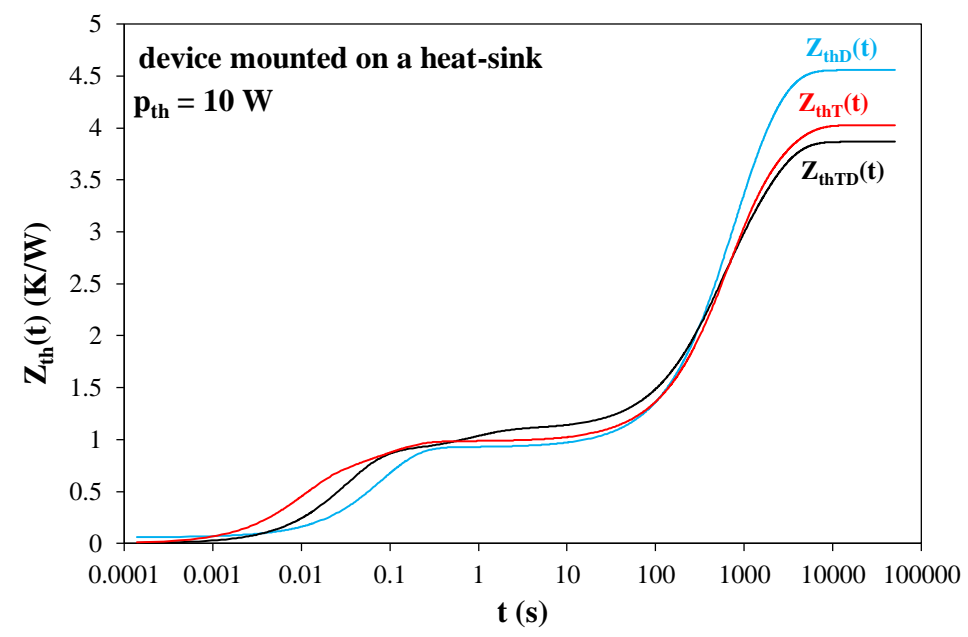

Figure 9. Measured waveforms of self and transfer transient thermal impedances in the investigated device situated on the heat-sink.

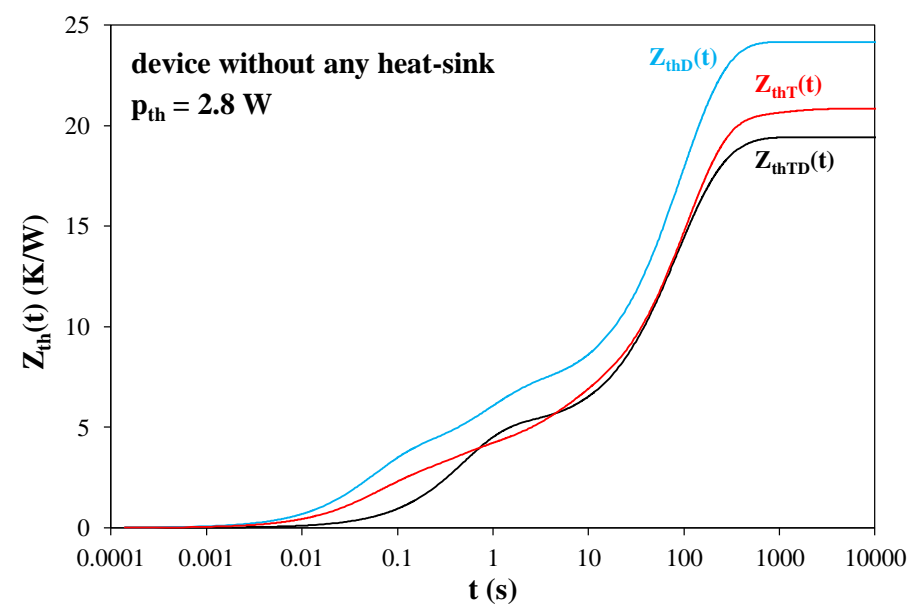

Figure 10. Measured waveforms of self and transfer transient thermal impedances in the tested device operating without any heat-sink.

As can be seen, waveforms of all the measured transient thermal impedances at fixed cooling conditions have similar shapes. The highest value of the measured waveforms is obtained for $Z_{t h D}(t)$. For the device operating on the heat-sink $Z_{t h T}(t)$ is lower by $12 \%$ than $Z_{t h D}(t)$, whereas for the device operating without any heat-sink the difference between the mentioned transient thermal impedances does not exceed $15 \%$. The lowest values were measured for transfer transient thermal impedance $Z_{\text {thTD }}(t)$.

It is proper to notice that waveforms of transient thermal impedances measured for the device situated on the heat-sink visibly differ from these waveforms measured for the transistor operating without any heat-sink. Particularly, one can see considerably longer set-up time of waveforms measured for the transistor situated on the heat-sink and the almost fivefold difference between their values measured at the steady state. Additionally, the thermally steady state is obtained after $200 \mathrm{~s}$ for the device without any heat-sink and over $2000 \mathrm{~s}$ for this device situated on the heat-sink. It is also proper to pay attention to the fact that, for the transistor situated on the heat-sink, for the first $100 \mathrm{~s}$ the measured values of transient thermal impedances do not exceed the catalogue value of thermal resistance between the semiconductor die and the case of the device $(1.7 \mathrm{~K} / \mathrm{W}$ for the diode and $0.77 \mathrm{~K} / \mathrm{W}$ for the transistor). This means that the heat-sink assures quasi-ideal cooling of the case of the device due to a high value of thermal capacitance of the heat-sink. In turn, for the device operating without any heat-sink, a continuous increase in the value of $Z_{t h}(t)$ is visible resulting from small thermal capacitance of the case of the investigated device. 
Waveforms of transient thermal impedances presented above were measured at fixed values of power dissipated in the investigated device. However, as has been shown (see, e.g., [18]), waveforms of transient thermal impedances of the considered transistor visibly change with changes in the value of ambient temperature and internal temperature of the investigated device. For example, changes in the value of thermal resistance at the change in the value of the internal temperature equal to $100 \mathrm{~K}$ can exceed even $20 \%$. As results from the measurements conducted by the authors and described in [18], the dependence of thermal capacitances on internal temperature is omittable, but the dependence of thermal resistance on this temperature is strong. Therefore, in the further part of this section, only internal temperatures of both dies $T_{j T}$ and $T_{j D}$ and the case temperature $T_{C}$ obtained at power dissipation only in the transistor are considered.

\subsection{Computed and Measured Values of Internal Temperature}

Values of internal temperatures of the dies included in the tested transistor operating in both types of considered cooling conditions are measured using the set-ups described in Section 3 and the case temperature of this transistor is measured with the use of the pyrometer Optex PT-3S. These temperatures are also computed with the use of the thermal model proposed in Section 2. The results of these measurements (points) and computations (solid lines) are shown in Figures 11 and 12. They were performed at fixed ambient temperature when power was dissipated in the IGBT operating in the active mode. In these figures, $T_{j T}$ describes the results of computations and measurements obtained with the use of $V_{G E}$ voltage as a thermo-sensitive parameter, while $T_{j D}$ corresponds to the measurements in which a thermo-sensitive parameter was voltage on the anti-parallel diode. The results of optical measurements are marked with $T_{C}$. In the case of optical measurements, the highest values of temperature on the surface of the case of the investigated transistor are registered.

Figure 11 illustrates the measured and computed dependences of internal temperatures of both dies and the case temperature of the investigated transistor situated on the heat-sink on heating power $P_{H}$ dissipated in this transistor.

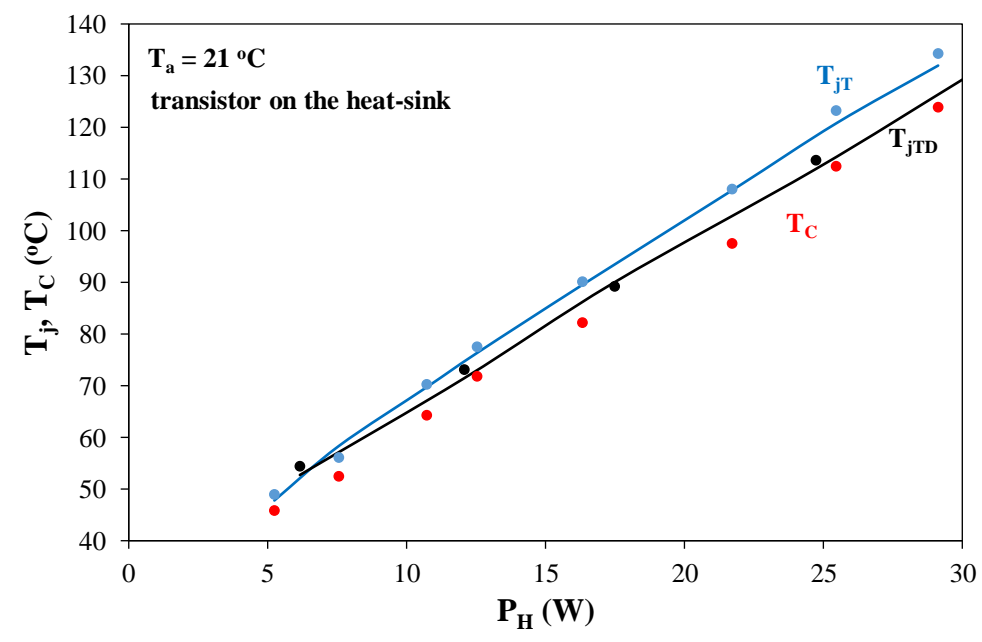

Figure 11. Measured and computed dependences of internal temperatures of the dies and the case temperature of the investigated device situated on the heat-sink on heating power dissipated in it at the steady state. 


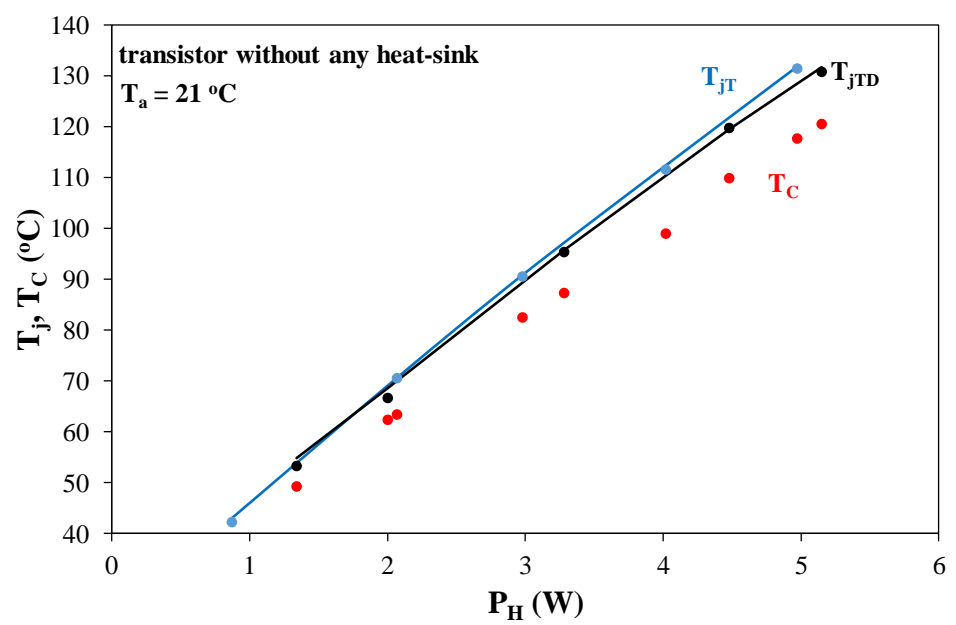

Figure 12. Measured and computed dependences of internal and case temperature of the investigated transistor operating without any heat-sink on heating power dissipated in the transistor.

As can be observed, all the considered dependences are increasing functions. Differences between values of the considered device increase together with an increase in power, attaining maximally $10{ }^{\circ} \mathrm{C}$ between the transistor internal temperature $T_{j T}$ and the case temperature $T_{C}$. The internal temperature of the diode $T_{j T D}$ is lower than temperature $T_{j T}$ by not more than $7^{\circ} \mathrm{C}$. The results of computations and measurements differ between each other by not more than $2{ }^{\circ} \mathrm{C}$.

In Figure 12, the dependences of the internal temperature and the case temperature of the investigated device operating without any heat-sink on heating power dissipated in the transistor are illustrated.

The obtained dependences of temperatures $T_{j T}, T_{j T D}$ and $T_{C}$ on heating power are monotonically increasing functions. Differences between the obtained values of temperatures $T_{j \mathrm{~T}}$ and $T_{j T D}$ are not high, and they do not exceed $3 \mathrm{~K}$. In turn, the measured values of the case temperature $T_{C}$ are lower than temperature $T_{j T}$ even by $10^{\circ} \mathrm{C}$. The results of computations fit well the results of measurements. Differences between these results do not exceed $2{ }^{\circ} \mathrm{C}$. Such small differences prove the correctness of the proposed thermal model of the considered devices.

As can be observed, good agreement between the results of measurements and modelling is obtained in a wide range of the internal temperature of both dies $\left(T_{j T}\right.$ and $\left.T_{j D}\right)$ from 40 to $>130^{\circ} \mathrm{C}$. The high border of this range is near the maximum allowable value of the internal temperature of this device, which is equal to $150{ }^{\circ} \mathrm{C}$ [26]. This means that the thermal mode proposed by us is usable in the whole range of the allowable internal temperature of the investigated device. Differences between the results of measurements and computations do not exceed the value of the measurement error.

In Figure 13, the waveforms of internal temperatures $T_{j T}$ and $T_{j D}$ computed with the use of the proposed model are shown. Figure 13a presents the results of investigations performed for the tested device operating without any heat-sink, whereas Figure 13b presents the results for the device situated on the heat-sink. The computations were performed for rectangular pulses trains of the duty cycle equal to 0.5 of power dissipated alternatively in the IGBT and in the diode. For the investigated device, the power dissipated in the transistor is shifted by half of the period in relation to the power dissipated in the diode. For the device without any heat-sink the amplitude of the dissipated power is equal to $4.5 \mathrm{~W}$ and the period is equal to $1000 \mathrm{~s}$, whereas for the device situated on the heat-sink this amplitude amounts to $25 \mathrm{~W}$ and the period is $2000 \mathrm{~s}$. 


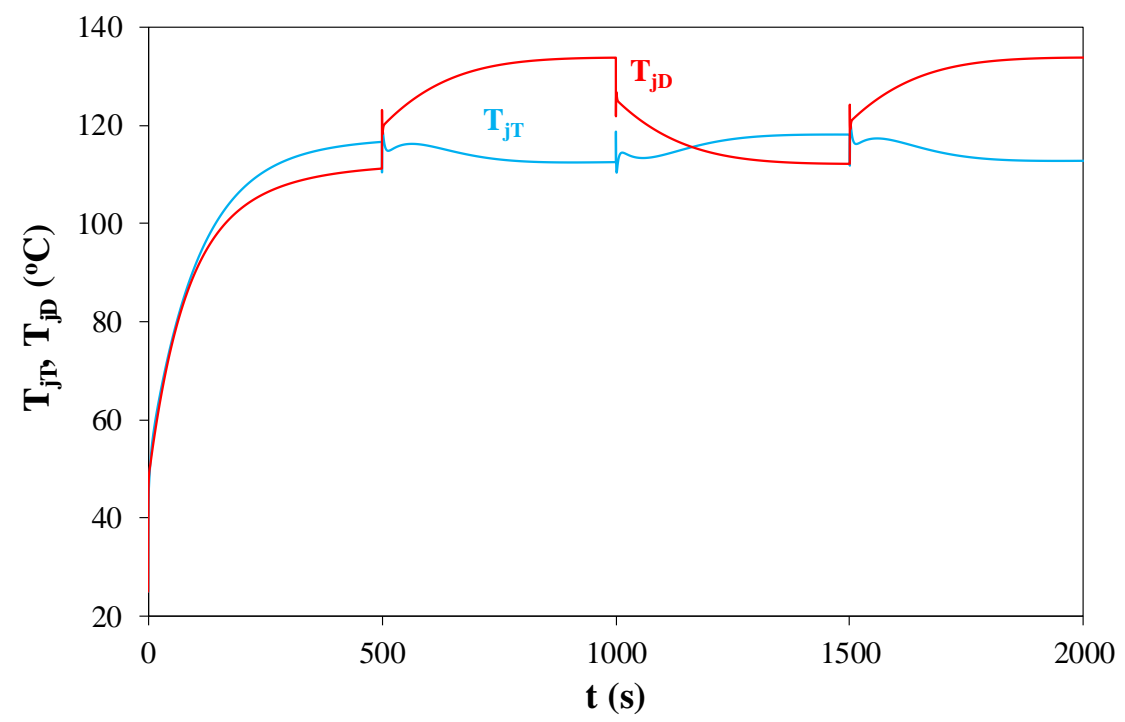

(a)

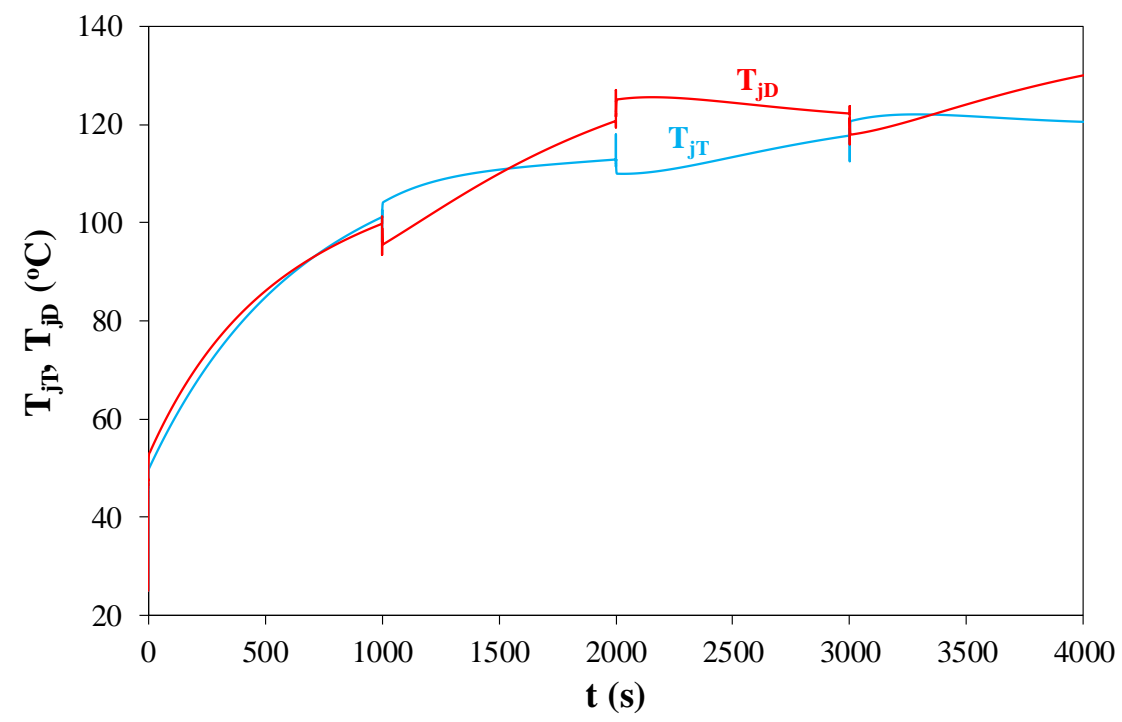

(b)

Figure 13. Computed waveforms of the internal temperature of the IGBT and the diode excited by the rectangular pulses train of power dissipated alternatively in these dies for the investigated devices: operating without any heat-sink (a); and situated on the heat-sink (b).

As can be observed, due to mutual thermal couplings, the internal temperature of the IGBT and the diode differ between each other by not more than by $20^{\circ} \mathrm{C}$. For the investigated device operating without any heat-sink for the period of time in which power is dissipated in the diode, temperature $T_{j D}$ is visibly higher than temperature $T_{j T}$. This is a result of the higher value of $R_{t h D}$ than $R_{t h T}$. When power is dissipated in the IGBT, temperature $T_{j T}$ exceeds temperature $T_{j D}$ after about $100 \mathrm{~s}$ after switching the power. In this case, the difference $T_{j T}-T_{j D}$ does not exceed $8{ }^{\circ} \mathrm{C}$.

Similar relations between the waveforms $T_{j T}(t)$ and $T_{j D}(t)$ can be observed for the investigated devices situated on the heat-sink. The obtained differences $T_{j D}-T_{j T}$ do not exceed $15^{\circ} \mathrm{C}$.

\section{Conclusions}

In the paper, the problem of effective modelling and measuring thermal properties of the power semiconductor device containing the IGBT and the diode in the common case 
is discussed. The nonlinear compact thermal model of these devices is proposed and the manner of estimating parameters of this model is described. This manner uses indirect electrical methods and different thermo-sensitive parameters.

On the basis of the obtained results, it is stated that thermometric characteristics $V_{G E}(T)$ are nonlinear and can be approximated with good accuracy by a square function. In turn, thermometric characteristics $V_{C E}(T)$ are practically linear. The slope of the characteristic $V_{G E}(T)$ is even twice bigger than the slope of the characteristic $V_{C E}(T)$. This means that with the use of voltage $V_{G E}$ it is possible to obtain a lower value of the measurement error of thermal resistance than with the use of voltage $V_{C E}$.

Using the considered electrical methods, self and transfer transient thermal impedances occurring in the thermal model of the power IGBT were measured for different cooling conditions of the investigated devices. Analysing the obtained results of measurements, it is easy to observe that transient thermal impedances $Z_{t h T}(t), Z_{t h T D}(t)$ and $Z_{t h D}(t)$ visibly differ from one another. Differences between these parameters at the steady state can reach even $15 \%$. This result shows that a thermal model of the IGBT containing dies of the transistor and the diode in the common case should take into account two internal temperatures of this device- one characterising internal temperature of the IGBT and the other internal temperature of the diode. Differences in the values of these temperatures can reach even $20^{\circ} \mathrm{C}$.

The obtained results of measurements of internal temperatures of the IGBT and the diode when power is dissipated in the IGBT only differ slightly between each other. These differences do not exceed $10^{\circ} \mathrm{C}$ due to a small difference between thermal resistance of the IGBT and transfer thermal resistance between the IGBT and the diode. Much bigger differences are observed if power is dissipated in the diode. The observed differences in the internal temperature of both dies contained in the investigated device prove that the highest temperature in this device can be observed in the diode. Therefore, it is very important to measure the internal temperature of both dies in order to verify that the investigated device operates in SOA. Additionally, the highest value of the device internal temperature affects its reliability.

The proposed compact thermal model makes it possible to more accurately model thermal properties of the investigated devices. The presented results of measurements and computations prove the usefulness of this model. The high accuracy of the proposed model in a wide range of dissipated power was obtained because the dependences of self and transfer thermal resistances on the device internal temperatures were taken into account. It is proved that the thermal model proposed in this paper is usable in the whole range of the allowable internal temperature of the investigated device.

In the article, only the thermal model of the IGBT co-packed with the diode is proposed. To perform simulations of circuits containing these two components, it is necessary to elaborate an electrothermal model of the considered device, which has to contain a thermal model and an electrical model of this device describing relationships between the device currents and voltages. The authors are currently working on such an electrothermal model.

Author Contributions: Conceptualisation, K.G. and P.G.; methodology, K.G. and P.G.; validation, P.G.; investigation, P.G.; writing—original draft preparation, K.G.; writing—review and editing, K.G. and P.G.; visualisation, K.G. and P.G.; and supervision, K.G. Both authors have read and agreed to the published version of the manuscript.

Funding: Project financed in the framework of the program by Ministry of Science and Higher Education called "Regionalna Inicjatywa Doskonałości" during the years 2019-2022, project number 006/RID/2018/19, the sum of financing 11,870,000 PLN.

Conflicts of Interest: The authors declare no conflict of interest.

\section{References}

1. Rashid, M.H. Power Electronic Handbook; Academic Press: New York, NY, USA; Elsevier: New York, NY, USA, 2007.

2. Perret, R. Power Electronics Semiconductor Devices; John Wiley \& Sons: Hoboken, NJ, USA, 2009. 
3. Kazimierczuk, M.K. Pulse-Width Modulated DC-DC Power Converters; Wiley: Chichester, UK, 2008.

4. Szymanski, J.R.; Zurek-Mortka, M.; Wojciechowski, D.; Poliakov, N. Unidirectional DC/DC converter with voltage inverter for fast charging of electric vehicle batteries. Energies 2020, 13, 4791. [CrossRef]

5. Hefner, A.R.; Blackburn, D.L. An analytical model for the steady-state and transient characteristics of the power insulated-gate bipolar transistor. Solid-State Electron. 1988, 31, 1513-1532. [CrossRef]

6. Górecki, P.; Górecki, K. Influence of thermal phenomena on dc characteristics of the IGBT. Int. J. Electron. Telecommun. 2018, 64, 71-76.

7. Hu, Z.; Zhang, W.; Wu, J. An improved electro-thermal model to estimate the junction temperature of IGBT module. Electronics 2019, 8, 1066. [CrossRef]

8. Górecki, K.; Górecki, P. Modelling dynamic characteristics of the IGBT with thermal phenomena taken into account. Microelectron. Int. 2017, 34, 160-164. [CrossRef]

9. Achiri, H.M.N.; Šmídl, V.; Peroutka, Z.; Streit, L. Least squares method for identification of IGBT thermal impedance networks using direct temperature measurements. Energies 2020, 13, 3749. [CrossRef]

10. Castellazzi, A.; Kraus, R.; Seliger, N.; Schmitt-Landsiedel, D. Reliability analysis of power MOSFET's with the help of compact models and circuit simulation. Microelectron. Reliab. 2002, 42, 1605-1610. [CrossRef]

11. Castellazzi, A.; Gerstenmaier, Y.C.; Kraus, R.; Wachutka, G.K.M. Reliability analysis and modeling of power MOSFETs in the 42-V-PowerNet. IEEE Trans. Power Electron. 2006, 21, 603-612. [CrossRef]

12. Liu, B.Y.; Wang, G.S.; Tseng, M.L.; Wu, K.J.; Li, Z.G. Exploring the electro-thermal parameters of reliable power modules: Insulated gate bipolar transistor junction and case temperature. Energies 2018, 11, 2371. [CrossRef]

13. Górecki, K.; Górecki, P.; Zarebski, J. Measurements of parameters of the thermal model of the IGBT module. IEEE Trans. Instrum. Meas. 2019, 68, 4864-4875. [CrossRef]

14. Chang, Y.; Li, W.H.; Luo, H.Z.; He, X.N.; Iannuzzo, F.; Blaabjerg, F.; Lin, W.X. A 3D thermal network model for monitoring imbalanced thermal distribution of press-pack IGBT modules in MMC-HVDC applications. Energies 2019, 12, 1319. [CrossRef]

15. Lasance, C.J.M.; Poppe, A. Thermal Management for LED Applications; Springer Science+Business Media: New York, NY, USA, 2014.

16. Bagnoli, P.; Casarosa, C.; Ciampi, M.; Dallago, E. Thermal resistance analysis by induced transient (TRAIT) method for power electronic devices thermal characterization. I. Fundamentals and theory. IEEE Trans. Power Electron. 1998, 13, 1208-1219. [CrossRef]

17. Avenas, Y.; Dupont, L.; Khatir, Z. Temperature measurement of power semiconductor devices by thermo-sensitive electrical parameters-A review. IEEE Trans. Power Electron. 2012, 27, 3081-3092. [CrossRef]

18. Górecki, K.; Górecki, P. Non-linear compact thermal model of the IGBT dedicated to SPICE. IEEE Trans. Power Electron. 2020, 35, 13420-13428. [CrossRef]

19. Oettinger, F.F.; Blackburn, D.L. Semiconductor Measurement Technology: Thermal Resistance Measurements; NIST/SP-400/86; U.S. Department of Commerce: Washington, DC, USA, 1990.

20. Zarębski, J.; Górecki, K. A method of measuring the transient thermal impedance of monolithic bipolar switched regulators. IEEE Trans. Compon. Packag. Technol. 2007, 30, 627-631. [CrossRef]

21. Dupont, L.; Avenas, Y. Preliminary evaluation of thermo-sensitive electrical parameters based on the forward voltage for online chip temperature measurements of IGBT devices. IEEE Trans. Ind. Appl. 2015, 51, 4688-4698. [CrossRef]

22. Dupont, L.; Avenas, Y.; Jeannin, P.-O. Comparison of junction temperature evaluations in a power IGBT module using an IR camera and three thermosensitive electrical parameters. IEEE Trans. Ind. Appl. 2013, 49, 1599-1608. [CrossRef]

23. Zeng, G.; Cao, H.; Chen, W.; Lutz, J. Difference in device temperature determination using p-n-Junction forward voltage and gate threshold voltage. IEEE Trans. Power Electron. 2019, 34, 2781-2793. [CrossRef]

24. JEDEC Standard JESD24-12. Thermal Impedance Measurement for Insulated Gate Bipolar Transistors—(Delta VCE(on) Method); JEDEC Solid State Technology Association: Arlington, VA, USA, 2004.

25. Górecki, P.; Górecki, K. Electrothermal averaged model of a diode-transistor switch including IGBT and a rapid switching diode. Energies 2020, 13, 3033. [CrossRef]

26. IRG4PC40UD. Insulated Gate Bipolar Transistor with Ultrafast Soft Recovery Diode, Data Sheet, International Rectifier. Available online: http:/ / www.irf.com/product-info/datasheets/data/irg4pc40ud.pdf (accessed on 15 January 2021).

27. Górecki, K.; Górecki, P. Investigations properties of selected methods of measurements of thermal parameters of the IGBT. In Proceedings of the 27th Internal Conference Mixed Design of Integrated Circuits and Systems Mixdes, Łódź, Poland, 25-27 June 2020; pp. 133-138.

28. Schweitzer, D.; Ender, F.; Hantos, G.; Szabó, P.G. Thermal transient characterization of semiconductor devices with multiple heat sources-Fundamentals for a new thermal standard. Microelectron. J. 2015, 46, 174-182. [CrossRef]

29. Szekely, V. A new evaluation method of thermal transient measurement results. Microelectron. J. 1997, 28, 277-292. [CrossRef]

30. Górecki, K.; Detka, K.; Górski, K. Compact thermal model of the pulse transformer taking into account nonlinearity of heat transfer. Energies 2020, 13, 2766. [CrossRef]

31. Janicki, M.; Napieralski, A. Considerations on electronic system compact thermal models in the form of RC ladders. In Proceedings of the 2019 IEEE 15th International Conference on the Experience of Designing and Application of CAD Systems (CADSM), Polyana, Ukraine, 26 February-2 March 2019.

32. Rencz, M.; Szekely, V. Measuring partial thermal resistances in a heat-flow path. IEEE Trans. Compon. Packag. Technol. 2002, 25, 547-553. [CrossRef] 
33. Górecki, K.; Zaręski, J.; Górecki, P.; Ptak, P. Compact thermal models of semiconductor devices-A review. Int. J. Electron. Telecommun. 2019, 65, 151-158.

34. Górecki, K.; Górecki, P. The Analysis of accuracy of selected methods of measuring the thermal resistance of IGBTs. Metrol. Meas. Syst. 2015, 22, 455-464. [CrossRef] 